(с) Н.В. Торопцова

ФГБНУ Научно-исследовательский институт ревматологии им. В.А. Насоновой, Москва, Россия

В статье представлен обзор с обсуждением клинических рекомендаций Американского колледжа ревматологов 2017 года по профилактике и лечению глюкокортикоидного остеопороза. Особенностью данной редакции рекомендаций является то, что в них представлены градации риска переломов не только у людей старше 40 лет, основанные на измерении минеральной плотности костей, подсчета 10-летней вероятности переломов по FRAX и наличию в анамнезе остеопоротических переломов, но и у лиц моложе 40 лет. В зависимости от уровня риска предложены рекомендации по инициации и длительности лечения в различных возрастных группах, также у детей с 4-х летнего возраста, у лиц после трансплантации органов и пациентов старше 30 лет, получающих очень высокие дозы глюкокортикоидов. Препаратами первого выбора эксперты называют пероральные бисфосфонаты, назначение которых предпочтительнее с учетом безопасности, стоимости и отсутствия доказательств по преимуществу других антиостеопоротических лекарственных средств для профилактики остеопоротических переломов. При непереносимости пероральных бисфосфонатов дана последовательность перехода на другие противоостеопоротические препараты. Рассматриваются вопросы применимости данных рекомендаций в отечественной врачебной практике.

КЛЮЧЕВЫЕ СЛОВА: глюкокортикоидный остеопороз; FRAX; лечение остеопороза; риск переломов; клинические рекомендации.

\title{
GLUCOCORTICOID-INDUCED OSTEOPOROSIS: PRESENTATION AND COMMENTS ON THE NEW AMERICAN COLLEGE OF RHEUMATOLOGY GUIDELINES
}

(C) Natalia V. Toroptsova

Research Institute of Rheumatology named after V.A. Nasonova, Moscow, Russia

The article presents a review of the clinical guidelines of 2017 American College of Rheumatology for prevention and treatment of glucocorticoid-induced osteoporosis. The guidelines i contain fracture risk gradation not only for people over 40 years, based on the measurement of bone mineral density, 10-year probability of fractures by FRAX and prior osteoporotic fractures, but also for people under 40 years. The guidelines present, recommendations for initial and follow-up treatment for prevention of glucocorticoid-induced osteoporosis according the level of risk of fractures in different age groups of adults, and in children from 4 years of age, in patients with organ transplant and patients older than 30 years, receiving very high-dose of glucocorticoids. Oral bisphosphonates were recommended as first line treatment due to safety, cost, and because of lack of evidence for superior antifracture benefits from other OP medications. Oral bisohosphonates could be switched to another medication in case of intolerance. The issues of applicability of these recommendations in national clinical practice are being discussed.

KEYWORDS: osteoporosis; glucocorticoid; fracture; practice guideline; comment.

\section{ВВЕДЕНИЕ}

Сегодня глюкокортикоиды (ГК), которые применяются в медицине с середины 20-го столетия, широко распространены для лечения различных воспалительных заболеваний, в том числе и системных ревматических болезней, благодаря противовоспалительному, иммунодепрессивному, противоаллергическому и другим свойствам. Их использование в клинической практике позволило не только улучшить качество жизни больных, но и значительно увеличить ее продолжительность. Однако длительный прием ГК может сопровождаться развитием серьезных неблагоприятных реакций, одной из которых является вторичный остеопороз (ОП), приводящий к возникновению низкоэнергетических переломов. При этом относительный риск переломов зависит как от суточной, так и кумулятивной дозы ГК. Так, van Staa T.P. и соавт. продемонстрировали, что если доза по преднизолону составляет 2,5 мг/сут, то риск перелома позвонка увеличен на 55\%, а при дозе 7,5 мг/сут уже на 518\% [1]. Кроме того, отмечалось еще более значимое увеличение риска переломов позвонков и бедра у пациентов, которые получают лечение ГК в высоких дозах - $\geq 30$ мг/сут с кумулятивной дозой более 5 г [2].

Несмотря на большое количество информации в периодической научной печати как по первичному, так и вторичному ОП, и, в частности, по глюкокортикоидному ОП (ГК-ОП), многие пациенты на ГК терапии как с уже произошедшим низкоэнергетическим переломом, так и без него, никогда не направлялись на денситометрическое исследование минеральной плотности костей (МПК) и/или не получали антиостеопоротических препаратов для профилактики последующих переломов $[3,4]$. 
Таблица 1. Критерии высокого, среднего и низкого риска переломов в зависимости от возраста у пациентов, получающих ГК [адаптировано из источника 5]

\begin{tabular}{|c|c|c|}
\hline $\begin{array}{c}\text { Риск } \\
\text { перелома }\end{array}$ & Взрослые люди $\geq 40$ лет & Взрослые люди до 40 лет \\
\hline \multirow{6}{*}{ Высокий } & Предшествующий остеопоротический перелом(ы) & \multirow{6}{*}{$\begin{array}{l}\text { Предшествующий остеопоротиче- } \\
\text { ский перелом(ы) }\end{array}$} \\
\hline & Т-критерий <-2,5СО в позвоночнике или бедре у мужчин $\geq$ 50лет & \\
\hline & и женщин в постменопаузе & \\
\hline & 10-летний риск основных остеопоротических переломов (алго- & \\
\hline & ритм $F R A X) \geq 20 \%$ & \\
\hline & 10-летний риск перелома бедра (алгоритм FRAX) 3\% & \\
\hline
\end{tabular}

10-летний риск основных остеопоротических переломов (алгоУмеренный ритм FRAX) 10\%-19\%

10-летний риск перелома бедра (алгоритм FRAX) >1\% - <3\%
МПК в поясничном отделе позвоночника или проксимальном отделе бедра по Z-критерию <-3СО или быстрая потеря МПК в течение 1 года $(\geq 10 \%)$

и

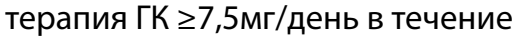
$\geq 6 \mathrm{mec}$

Нет вышеперечисленных факторов, кроме ГК-терапии

Низкий

10-летний риск основных остеопоротических переломов (алгоритм FRAX) $<10 \%$

10-летний риск перелома бедра (алгоритм FRAX) $\leq 1 \%$

\section{РЕКОМЕНДАЦИИ ПО ГК-ОП АМЕРИКАНСКОГО КОЛЛЕДЖА РЕВМАТОЛОГОВ (ACR)}

В 1996 году были выпущены первые рекомендации по ГК-ОП Американского колледжа ревматологов (ACR), которые были дополнены и переизданы в 2001 и 2010 годах. В 2017 году ACR представил новые клинические рекомендации по профилактике и лечению ГК-ОП, разработанные с использованием методологии Grading of Recommendations Assessment, Development and Evaluation (GRADE). Они делятся на настоятельные (строгие, высокой силы доказательности) и условные (умеренной степени доказательности). Настоятельная рекомендация свидетельствует об уверенности группы разработчиков в том, что положительные последствия рекомендации превышают возможные нежелательные эффекты, поэтому она применима ко всем или почти всем пациентам, и только небольшая доля больных не сможет или не захочет следовать рекомендации. Условная - (рекомендация умеренной силы) означает, что группа разработчиков считает, что положительные последствия этой рекомендаций, с большой долей вероятности перевешивают нежелательные эффекты, поэтому будут применяться к большинству пациентов, но некоторые не смогут или не захотят следовать им [5]. Раздел лечения ГК ОП включает лекарственные препараты, которые были одобрены Управлением по контролю качества пищевых продуктов и лекарственных препаратов США (FDA) до 2015 года [5].

Положительным моментом в данных рекомендациях является то, что в них представлена градация риска переломов (низкий, умеренный и высокий) не только для женщин в постменопаузе и мужчин 50 лет и старше, но предложены критерии для людей моложе 40 лет (табл. 1).

В старшей возрастной группе пациентов при использовании алгоритма FRAX градации риска основаны на экономических расчетах, специфичных для США и рекомендованных Национальным фондом ОП [6]. Авторы предлагают использовать поправочные коэффициенты в зависимости от дозы в пересчете на преднизолон при определении абсолютного риска по FRAX, аналогичные предложенным Kanis J.A. и соавт. [7]. Так, при дозе ГК более 7,5мг/сут в пересчете на преднизолон поправочный коэффициент для основных остеопоротических переломов составляет 1,15, а для перелома бедра - 1,2. При этом дозы ГК 2,5 - 7,5 мг/сут пересчета не требуют. Аналогичный принцип расчета 10-летней вероятности перелома у пациентов, получающих ГК, предложен и Российской ассоциацией по остеопорозу [8]. Высокий риск переломов диагностируется в этой возрастной группе также и при наличии в анамнезе остеопоротического перелома, и при снижении МПК более, чем на 2,5 стандартных отклонений (СО) в позвоночнике и бедре.

У пациентов на ГК терапии моложе 40 лет алгоритм FRAX не применим, поэтому авторами американских рекомендаций было предложено взрослых людей с остеопоротическим переломом в анамнезе рассматривать как имеющих высокий риск повторных переломов, что не противоречит Европейским и Российским рекомендациям. Кроме того, даны градации умеренного и низкого риска для данной возрастной группы (см. табл. 1), на основании которых разработаны рекомендации по профилактике ОП и переломов. В предшествующих рекомендациях 2010г. тактика ведения женщин в пременопаузе и мужчин моложе 50 лет зависела только от наличия или отсутствия предшествующего перелома, при этом низкая МПК не являлась основанием для назначения антиостеопоротической терапии ввиду недостаточности данных по профилактике переломов на фоне лечения в этой возрастной группе.

По рекомендациям ACR оценка 10-летней вероятности перелома по FRAX (https://www.shef.ac.uk/FRAX) у пациентов 40 лет и старше должна проводиться как можно раньше, по крайней мере в пределах первых 6 месяцев от начала терапии, с поправкой на дозу ГК и с включением показателя МПК шейки бедра или без него (при невозможности проведения денситометрии). Для лиц моложе 
Таблица 2. Рекомендации по инициации лечения для профилактики ГК-ОП в зависимости от риска перелома у пациентов 40 лет и старше, начавщих длительную терапию ГК [адаптировано из источника 5].

Низкий риск остеопороти- Оптимизировать потребление кальция и витамина D и модифицировать стиль жизни ческих переломов предпочтительнее лечения БФ, терипаратидом, деносумабом или ралоксифеном. Лечение пероральными БФ предпочтительнее приему только кальция и витамина $D$ Лечение пероральными БФ предпочтительнее терапии внутривенными БФ, терипаратидом, деносумабом или ралоксифеном.

Пероральные БФ предпочтительнее с учетом их безопасности, стоимости и отсутствия доказанных преимуществ других остеопоротических препаратов для профилактики остеопоротических переломов.

Умеренный риск остеопоротических переломов

Если имеется непереносимость пероральных БФ, то последовательность назначения других препаратов:

$$
\begin{aligned}
& \text { - внутривенные БФ (более высокий риск НР по сравнению с пероральными БФ) } \\
& \text { - терипаратид (стоимость и бремя терапии ежедневными инъекциями) } \\
& \text { - деносумаб (отсутствие данных о безопасности у людей, получавших иммуноде- } \\
& \text { прессанты) }
\end{aligned}
$$

- ралоксифен (для женщин в постменопаузе, у которых ни одно из перечисленных выше лекарств не является подходящим). Отсутствие адекватных данных о преимуществах (воздействие на риск переломов позвонков и бедер у пациентов с ГК) и потенциальный вред (риск повышения свертывания крови и смертности).

Лечение пероральными БФ предпочтительнее приему только кальция и витамина $D$

Лечение пероральными БФ предпочтительнее терапии внутривенными БФ, терипаратидом, деносумабом или ралоксифеном.

Пероральные БФ предпочтительнее с учетом безопасности, стоимости и отсутствия доказательств преимущества других препаратов для профилактики остеопоротических переломов.

Если имеется непереносимость пероральных БФ, то последовательность назначения

Высокий риск остеопоротических переломов других препаратов:
- внутривенные БФ (более высокий риск НР) по сравнению с пероральными БФ
- терипаратид (стоимость и бремя терапии ежедневными инъекциями)
- деносумаб (отсутствие данных о безопасности у людей, получавших иммуноде- прессанты)
- ралоксифен (для женщин в постменопаузе, у которых ни одно из перечислен- ных выше лекарств не является подходящим). Отсутствие адекватных данных о преимуществах (воздействие на риск переломов позвонков и бедер у пациентов с ГК) и потенциальный вред (риск повышения свертывания крови и смертности).

40 лет денситометрическое исследование аксиальных отделов скелета должно быть сделано в течение первых 6 месяцев от начала терапии ГК, особенно если у пациента в анамнезе есть низкоэнергетический перелом или другие значимые клинические факторы риска. В последующем клиническая оценка риска переломов должна осуществляться каждые 12 месяцев, если лечение ГК продолжается. На денситометрическое обследование следует направлять больных каждые 2-3года, при этом более рано тех пациентов, которые принимают очень высокие дозы ГК, имеют в анамнезе остеопоротические переломы или низкую МПК. Реже могут оцениваться пациенты, находящиеся на низких дозах ГК и не имеющие других факторов риска ОП.

Все пациенты, получающие ГК в дозе $\geq 2,5$ мг/сут в течение $\geq 3$ месяцев должны оптимизировать потребление кальция (1000-1200 мг/сут) и витамина D (600-800 ME/сут). Согласно Российским клиническим рекомендациям доза кальция аналогична, а профилак- тическая доза витамина D должна составлять не менее 800-1000ME и может достигать 2000MЕ/сут при наличии выявленного ОП и приема антирезорбтивных препаратов [9]. Кроме того, следует модифицировать стиль жизни, компонентами которого являются сбалансированное питание, оптимизация массы тела, отказ от курения, регулярные физические упражнения с нагрузкой весом и сопротивлением, ограничение потребления алкоголя. Это условные рекомендации по методологии GRADE.

В дальнейшем рекомендации по лечению ОП даются в зависимости от возраста и риска переломов.

Профилактика и лечение ОП и ОП переломов у пациентов 40 лет и старше представлена в таблице 2 . При низком риске переломов рекомендуется только оптимизировать потребление кальция и витамина D, а также изменить стиль жизни, как и для всех лиц, у которых лечение ГК планируется продолжать 3 и более месяцев (рекомендация средней силы для приема кальция 
Таблица 3. Рекомендации по инициации лечения для профилактики ГК-ОП в зависимости от риска перелома у пациентов моложе 40 лет, начавших длительную терапию ГК [адаптировано из источника 5].

\begin{tabular}{|c|c|}
\hline Низкий риск перелома & Средний и высокий риск перелома \\
\hline \multirow{7}{*}{$\begin{array}{l}\text { Оптимизировать } \\
\text { потребление кальция } \\
\text { и витамина D } \\
\text { и модифицировать } \\
\text { стиль жизни } \\
\text { предпочтительнее } \\
\text { лечения БФ, } \\
\text { терипаратидом, } \\
\text { деносумабом. }\end{array}$} & Лечение пероральными БФ предпочтительнее приему только кальция и витамина D \\
\hline & $\begin{array}{l}\text { Лечение пероральными БФ предпочтительнее терапии внутривенными БФ, терипара- } \\
\text { тидом, деносумабом }\end{array}$ \\
\hline & $\begin{array}{l}\text { Пероральные БФ предпочтительнее с учетом безопасности, стоимости и отсутствию } \\
\text { доказательств по преимуществу других препаратов для профилактики остеопоротиче- } \\
\text { ских переломов }\end{array}$ \\
\hline & $\begin{array}{l}\text { Если имеется непереносимость пероральных БФ, то последовательность назначения } \\
\text { других препаратов: }\end{array}$ \\
\hline & - Внутривенные БФ (более высокий риск НР) по сравнению с пероральными БФ \\
\hline & - Терипаратид (стоимость и бремя терапии ежедневными инъекциями) \\
\hline & $\begin{array}{l}\text { - Деносумаб (отсутствие данных о безопасности у людей, получавших } \\
\text { иммунодепрессанты) }\end{array}$ \\
\hline
\end{tabular}

и витамина D по сравнению с пероральными БФ, терипаратидом и деносумабом и настоятельная рекомендация для них по сравнению с внутривенными БФ и ралоксифеном, т.е. в данной ситуации самое существенное оптимизировать потребление кальция и витамина D и модифицировать образ жизни).

Для пациентов с умеренным и высоким риском следует принимать антиостеопоротические препараты, при этом лечение надо начинать с пероральных бисфосфонатов (БФ), а при их непереносимости или плохой приверженности следует перейти на другой препарат в следующей последовательности: внутривенный БФ, терипаратид, деносумаб. Для женщин в постменопаузе возможно назначение ралоксифена, однако нет доказательств его эффективности при ГК-ОП, поэтому его назначение должно рассматриваться только при наличии противопоказаний для терапии другими препаратами (настоятельная рекомендация для пероральных БФ по сравнению с приемом только кальция и витамина $D$ у пациентов с высоким риском, все остальные рекомендации условные как при высоком, так и умеренном риске перелома).

Профилактика и лечение ОП и остеопоротических переломов у пациентов моложе 40 лет представлена в таблице 3. Показанием для назначения как у женщин, при отсутствии вероятности деторождения, так и у мужчин является наличие перелома в анамнезе, или прием ГK >6 месяцев в дозе $\geq 7,5 \mathrm{Mr/день} \mathrm{и} \mathrm{Z-критерий} \mathrm{<-3СО}$ или потеря МПК в любой зоне более 10\% в год. Этим пациентам должны назначаться пероральные БФ. При их непереносимости рекомендуются другие антиостеопоротические препараты в следующей очередности: внутривенные БФ, терипаратид, деносумаб, как и у лиц старше 40 лет. Ралоксифен у женщин в пременопаузе и у мужчин не применяется (условные рекомендации из-за низкого и очень низкого качества доказательств влияния на абсолютный риск переломов и косвенных и низкого качества доказательств относительного вреда и преимуществ альтернативных препаратов в этой возрастной группе).

В рекомендациях выделены особые популяции больных, получающие длительно лечение ГК - это женщины детородного возраста, имеющие средний или высокий риск остеопоротических переломов; лица $\geq 30$ лет, которые получают очень высокие дозы ГК (начальная доза по преднизону $\geq 30$ мг/сут или кумулятивная доза $\geq 5$ г в течение года); пациенты после пересадки органов; дети от 4 до 17 лет (табл. 4). Все рекомендации для этих групп больных являются условными из-за косвенных и очень низкого качества доказательств преимуществ и вреда этих препаратов как для плода во время беременности у женщин в пременопаузе, так и их влияния на абсолютный риск переломов у молодых людей, лиц после трансплантации органов и детей. Для детей имеются умеренно-качественные доказательства низкого вреда пероральных БФ и их меньшего потенциального вреда по сравнению с внутривенными БФ.

В рекомендациях дано определение неэффективности начатой антиостеопоротической терапии у больных старше 40 лет - это произошедший перелом через 18 месяцев и более после начала терапии пероральными БФ или снижение МПК на 10\% и более за год. В этих случаях следует перевести пациента на другой класс препаратов (терипаратид или деносумаб) или на внутривенный $Б Ф$, если неэффективность связана с плохой приверженностью или плохой всасываемостью в желудочно-кишечном тракте перорального БФ. Это является предпочтительней, чем прием только кальция и витамина D. Авторы отметили эти рекомендации как условные из-за отсутствия доказательств преимущества одного из препаратов над другими.

Больным старше 40 лет, которые в течение 5-лет получали пероральные БФ, но продолжают лечение ГК и имеют умеренный или высокий риск переломов, не следует останавливать антиостеопоротическую терапию, принимая ее совместно с препаратами кальция и витамина D, что является более предпочтительным, чем перевод больных только на препараты кальция и витамина D. Предлагается продолжить прием пероральных БФ до 7-10 лет или перевести на внутривенный БФ при непереносимости или плохой приверженности таблетированному БФ. Возможен перевод также на другой класс антиостеопоротических препаратов (терипаратид или деносумаб). Следует рассматривать вероятность редких осложнений при длительном лечении антирезорбтивными препаратами, которые включа- 
Таблица 4. Рекомендации по инициации лечения в особых популяциях пациентов, начавших длительную терапию ГК [адаптировано из 5]

Лечение пероральными БФ предпочтительнее приему только препаратов кальция и витамина D, терипаратида, внутривенных БФ или деносумаба.

Женщины детородного возраста, имеющие умеренный или высокий риск переломов, которые не планируют беременность в течение периода лечения ОП и используют эффективные средства контрацепции или не имеющие половых контактов
Пероральные БФ предпочтительнее с учетом безопасности, стоимости и отсутствия доказательств по преимуществу других препаратов для предотвращения остеопоротических переломов.

Если имеется непереносимость или невозможность назначения пероральных БФ, то последовательность назначения других препаратов:

\section{- Терипаратид (безопасность, стоимость и бремя терапии ежедневными инъек- циями)}

Рассматривать назначение других препаратов только в группах пациентов высокого риска ввиду отсутствия данных о безопасности использования этих лекарств во время беременности:

- Внутривенные БФ (потенциальный риск для развития плода при внутривенном введении БФ во время беременности)

- Деносумаб (потенциальный риск для развития плода во время беременности)
Пациенты $\geq 30$ лет, которые получают очень высокие дозы ГК (начальная доза преднизона $\geq 30$ мг/сут или кумулятивная доза $\geq 5$ г в течение года)

Пациенты после пересадки органов, клубочковой фильтрацией $\geq 30$ лл/мин и без признаков метаболических заболеваний костей, которые продолжают терапию ГК

\section{Дети в возрасте от 4} до 17 лет, получающие ГК $\geq 3$ месяца

Дети в возрасте от 4 до 17 лет с переломами в анамнезе, получающие ГК 3 месяца и более в дозе $\geq 0,1 \mathrm{мг/кг/сут}$
Лечение пероральными БФ предпочтительнее приему только кальция и витамина D.

Лечение пероральными БФ предпочтительнее терапии внутривенными БФ, терипаратидом или деносумабом.

Пероральные БФ предпочтительнее с учетом безопасности, стоимости и отсутствия доказательств преимущества других препаратов для предотвращения остеопоротических переломов.

Если имеется непереносимость или невозможность назначения пероральных БФ, то последовательность назначения других препаратов указана в зависимости от возраста в таблицах 2 и 3.

Лечить в соответствии с рекомендациями в зависимости от возраста у пациентов без трансплантации органов, с дополнительными рекомендациями:

- Оценка состояния специалистом по метаболическим заболеваниям костей рекомендуется для всех пациентов с трансплантацией почек

- Не рекомендуется применять деносумаб из-за отсутствия адекватных данных по его безопасности при инфекциях у взрослых, получающих несколько иммунодепрессантов.

Оптимизировать потребление кальция (1000мг/сут) и витамина D (600ME/сут), модифицировать стиль жизни.

Лечение пероральными БФ (внутривенными БФ, если есть противопоказания лечению пероральными БФ) плюс кальций и витамин D предпочтительнее терапии только кальцием и витамином D. ют риск остеонекроза челюсти и атипичных переломов (условные рекомендации из-за очень низкого качества данных о преимуществах и вреде продолжения лечения у пациентов, получавших ГК, и среднего качества данных у пациентов с первичным ОП по поводу преимущества и вреде продолжения лечения пероральными БФ после 5 лет терапии).

Антиостеопоротическое лечение после отмены ГК у пациентов в возрасте $\geq 40$ лет рекомендуется прекратить, если на этот момент риск перелома у них низкий. Однако прием препаратов кальция и витамина D следует продолжить. Если риск перелома остается высоким, то терапию ОП останавливать не надо (настоятельная рекомендация, основанная на мнении экспертов).

\section{ОБСУЖДЕНИЕ}

По нашему мнению, в данной редакции рекомендаций ACR по ГКОП имеются некоторые моменты, которые могут осложнить понимание практикующими врачами, когда и кого начинать лечить. Это связано с тем, что деление на группы построено по возрастному принципу старше и моложе 40 лет, что, наверное, обусловлено тем, что подсчет абсолютного 10-летнего риска переломов по FRAX осуществляется у людей от 40 до 90 лет. В тоже время следует помнить, что рассматривая женщину от 40 до 50-55 лет, как кандидата для профилактики остеопоротических переломов, необходимо всегда задавать вопрос имеет ли женщина потенциал к деторождению или нет, так как от этого зависит выбор лекарственного 
препарата. В предшествующих версиях рекомендаций ACR по ГКОП, так же, как и в Российских, женщины были разделены в зависимости от наличия или отсутствия у них менопаузы, что является более логичным. Кроме того, алгоритм FRAX может применяться только у женщин в возрасте 40 лет и старше, находящихся в постменопаузе, и у мужчин старше 50 лет, что связано со сбором эпидемиологических данных по переломам, лежащих в его основе [10].

Для практикующих врачей в России может быть интересным, а возможно ли использовать критерии риска переломов, которые применяются в США. В настоящее время этот вопрос остается открытым, так как не проводилось исследований по оценке чувствительности и специфичности их порогов у наших соотечественников. В тоже время следует помнить, что они основаны на экономических расчетах, специфичных для США. В нашей стране для определения высокого риска переломов используется возрастзависимая кривая, рассчитанная по алгоритму FRAX, разработанному для нашей страны на основании российских эпидемиологических данных по низкоэнергетическим переломам. Высокий абсолютный риск перелома у конкретного пациента диагностируется при значениях FRAX выше порога вмешательства, соответствующего возрасту больного, а антиостеопоротическое лечение может быть назначено без проведения денситометрического обследования. Для лиц, принимающих длительно ГК следует использовать поправочные коэффициенты в зависимости от дозы ГК. Показаниями для назначения терапии у женщин в постменопаузе и мужчин в возрасте 50 лет и старше, которым проводится или планируется терапия ГК в течение трех и более месяцев, также является любой из следующих критериев: перелом при минимальной травме в анамнезе, возраст старше 70 лет, прием высоких доз ГК ( $\geq 7,5$ мг/сут), МПК по Т-критерию $\leq-1,5$ СО в аксиальных отделах скелета [8].

Впервые в последней редакции рекомендаций ACR была представлена градация риска у молодых людей и детей, что является оправданным, так как в предыдущих версиях назначение антиостеопоротического лечения было показано только у лиц с перенесенным низкоэнергетическим переломом, а при его отсутствии рекомендовалось только оптимизировать потребление кальция и витамина D. B тоже время, всегда остается тревога за пациентов, у которых перелом еще не случился, но были низкая МПК и имелись клинические факторы риска. В наших рекомендациях решение о назначении терапии у пациентов этой возрастной группы с МПК по Z-критерию $\leq-2$ СО отдано на рассмотрение лечащего врача, однако также указано, что доказательной базы о необходимости назначения терапии нет. Препаратами выбора у молодых пациентов и детей являются пероральные БФ, однако отсутствие данных об отсроченных результатах лечения ОП в этих возрастных группах, может привести как недостаточному, так и чрезмерному их использованию. Поэтому в настоящее время существует необходимость в проведении дальнейших исследований по изучению результатов применения антиостеопоротических препаратов у этой категории больных.
Следует заметить, что среди лекарственных средств нет упоминания о применении активных метаболитов витамина D для лечения и профилактики ГК ОП. В тоже время мета-анализ рандомизированных клинических исследований продемонстрировал, что они имеют большую эффективность в предотвращении потери МПК и уменьшении риска переломов позвонков по сравнению не только с плацебо или отсутствием лечения, но и с нативным витамином D или монотерапией препаратами кальция, при этом данные относительно профилактики переломов относятся только к приему альфакальцидола [11]. В отечественных клинических рекомендациях активные метаболиты являются препаратами второго выбора у больных с ГКОП [8].

В рассматриваемых рекомендациях препаратом первого выбора являются пероральные БФ, с которых следует начинать профилактику и лечение ГК ОП. Авторы это связывают с тем, что они безопасны и предпочтительнее с учетом их стоимости и отсутствия доказательств по преимуществу других препаратов для профилактики остеопоротических переломов. В 2016 году вышел мета-анализ по оценке эффективности лечения ГК ОП различными антиостеопоротическими препаратами, который показал, что наиболее выраженный эффект в предотвращении переломов позвонков был у терипаратида, а затем следовали ризедронат и золедроновая кислота. Для профилактики периферических переломов - терипаратид, а затем ризедронат [12]. Деносумаб в настоящее время не зарегистрирован для лечения ГКОП, поэтому его применение ограничено - только у женщин в постменопаузе и мужчин старше 50 лет при непереносимости или невозможности использования других антиостеопоротических препаратов.

\section{ЗАКЛЮЧЕНИЕ}

В заключение хочется отметить, что цель антиостеопоротического лечения больных, получающих длительно ГК, является снижение риска низкоэнергетических переломов, а первый шаг к этому - своевременная коррекция дозы ГК до минимально терапевтически эффективной. Пациенты, принимающие ГК, требуют диспансерного наблюдения по контролю антиостеопоротического лечения и, при доступности, проведения денситометрического обследования. Отмена ГК дает возможность остановить прием патогенетического лечения ОП, однако следует продолжить прием препаратов кальция и витамина D и контролировать МПК. У пожилых пациентов с высоким риском переломов следует продолжать антиостеопортическое лечение, так как у них выходят на первый план механизмы потери костной массы, связанные с процессом старения.

\section{ДОПОЛНИТЕЛЬНАЯ ИНФОРМАЦИЯ}

Конфликт интересов. Автор декларирует отсутствие явных и потенциальных конфликтов интересов, связанных с публикацией настоящей статьи. 


\section{СПИСОК ЛИТЕРАТУРЫ | REFERENCES}

1. van Staa TP, Leufkens HGM, Abenhaim L, et al. Oral corticosteroids and fracture risk: relationship to daily and cumulative doses. Rheumatology (Oxford). 2000;39(12):1383-1389. doi: 10.1093/rheumatology/39.12.1383

2. van Staa TP, Geusens P, Pols HA, et al. A simple score for estimating the long-term risk of fracture in patients using oral glucocorticoids. QJM. 2005;98(3):191-198. doi: 10.1093/qjmed/hci029

3. Curtis JR, Westfall AO, Allison JJ, et al. Longitudinal patterns in the prevention of osteoporosis in glucocorticoid-treated patients. Arthritis Rheum. 2005;52(8):2485-2494. doi: 10.1002/art.21194

4. Баранова И.А., Ершова О.Б., Анаев Э.Х., и др. Анализ оказания консультативной медицинской помощи пациентам с глюкокортикоидным остеопорозом или риском его развития по данным анкетирования пациентов (исследование ГЛЮКОСТ). // Терапевтический архив. - 2015. - Т. 87. - №5. - С. 58-64. [Baranova IA, Ershova OB, Anaev EK, et al. Analysis of the state-of-the-art of consulting medical care to patients with glucocorticoid-induced osteoporosis or its risk according to the data of a questionnaire survey (GLUCOST study). Ter Arkh. 2015;87(5):58-64. (In Russ.)] doi: 10.17116/terarkh201587558-64

5. Buckley L, Guyatt G, Fink HA, et al. 2017 American College of Rheumatology Guideline for the Prevention and Treatment of Glucocorticoid-Induced Osteoporosis. Arthritis Rheumatol. 2017:69(8):1521-1537. doi: 10.1002/art.40137

6. Dawson-Hughes B, Tosteson AN, Melton $\sqcup$, 3rd, et al. Implications of absolute fracture risk assessment for osteoporosis practice guidelines in the USA. Osteoporos Int. 2008;19(4):449-458. doi: 10.1007/s00198-008-0559-5

7. Kanis JA, Johansson H, Oden A, McCloskey EV. Guidance for the adjustment of FRAX according to the dose of glucocorticoids. Osteoporos Int. 2011;22(3):809-816. doi: 10.1007/s00198-010-1524-7
8. Баранова И.А., Торопцова Н.В., Лесняк О.М. Основные положения клинических рекомендаций «Диагностика, профилактика и лечение глюкокортикоидного остеопороза у мужчин и женщин 18 лет и старше». // Остеопороз и остеопатии. - 2014. - Т. 17. №3. - C. 34-37. [Baranova IA, Toroptsova NV, Lesnyak OM. The Main Provisions of the Guidelines «Diagnosis, Prevention and Treatment of Glucocorticoid Osteoporosis in Men and Women 18 Years and Older». Osteoporosis and bone diseases. 2014;17(3):34-37. (In Russ.)] doi: 10.14341/osteo2014334-37

9. Белая Ж.Е., Белова К.Ю., Бордакова Е.В., и др. Профилактика, диагностика и лечение дефицита витамина D и кальция среди взрослого населения и у пациентов с остеопорозом. В кн.: Рекомендации Российской ассоциации по остеопорозу. / Под ред. Лесняк О.М. - М.: ГЭОТАР-Медиа; 2016. [Belaya ZE, Belova KY, Bordakova EV, et al. Profilaktika, diagnostika i lechenie defitsita vitamina $D$ i kal'tsiya sredi vzroslogo naseleniya i u patsientov s osteoporozom. In: Lesnyak OM, editor. Rekomendatsii Rossiyskoy assotsiatsii po osteoporozu. Moscow: GEOTAR-Media; 2016. (In Russ.)]

10. Kanis JA, McCloskey EV, Johansson H, et al. Development and use of FRAX in osteoporosis. Osteoporos Int. 2010;21 Suppl 2:S407-413. doi: 10.1007/s00198-010-1253-y

11. de Nijs RN, Jacobs JW, Algra A, et al. Prevention and treatment of glucocorticoid-induced osteoporosis with active vitamin D3 analogues: a review with meta-analysis of randomized controlled trials including organ transplantation studies. Osteoporos Int. 2004;15(8):589-602. doi: 10.1007/s00198-004-1614-5

12. Amiche MA, Albaum JM, Tadrous M, et al. Efficacy of osteoporosis pharmacotherapies in preventing fracture among oral glucocorticoid users: a network meta-analysis. Osteoporos Int. 2016;27(6):1989-1998. doi: 10.1007/s00198-015-3476-4

\section{ИНФОРМАЦИЯ ОБ АВТОРАХ [AUTHORS INFO]}

Торопцова Наталья Владимировна, д.м.н. [Natalia V. Toroptsova, MD, PhD]; адрес: 115522, Россия, г. Москва, Каширckoe шосce, 34A [address: 34A, Kashirskoe Shosse, Moscow, 115522 Russia]; ORCID: http://orcid.org/0000-0003-4739-4302; eLibrary SPIN: 5650-2058; e-mail: torop@irramn.ru

\section{ЦИТИРОВАТЬ:}

Торопцова Н.В. Глюкокортикоидный остеопороз: представление и обсуждение новых клинических рекоменда-ций Американского колледжа ревматологов // Остеопороз и остеопатии. — 2017. — Т. 20. — №3. — С.114-120. doi: 10.14341/osteo20173114-120

\section{TO CITE THIS ARTICLE:}

Toroptsova NV. Glucocorticoid-induced osteoporosis: presentation and comments on the new American College of Rheuma-tology guidelines. Osteoporosis and bone diseases. 2017;20(3):114-120. doi: 10.14341/osteo20173114-120 